\title{
Frailty trajectories to identify end of life: a longitudinal population-based study
}

\author{
Daniel Stow, Fiona E. Matthews and Barbara Hanratty ${ }^{*}$ (D)
}

\begin{abstract}
Background: Timely recognition of the end of life allows patients to discuss preferences and make advance plans, and clinicians to introduce appropriate care. We examined changes in frailty over 1 year, with the aim of identifying trajectories that could indicate where an individual is at increased risk of all-cause mortality and may require palliative care.

Methods: Electronic health records from 13,149 adults (cases) age 75 and over who died during a 1-year period (1 January 2015 to 1 January 2016) were age, sex and general practice matched to 13,149 individuals with no record of death over the same period (controls). Monthly frailty scores were obtained for 1 year prior to death for cases, and from 1 January 2015 to 1 January 2016 for controls using the electronic frailty index (eFl; a cumulative deficit measure of frailty, available in most English primary care electronic health records, and ranging in value from 0 to 1). Latent growth mixture models were used to investigate longitudinal patterns of change and associated impact on mortality. Cases were reweighted to the population level for tests of diagnostic accuracy.

Results: Three distinct frailty trajectories were identified. Rapidly rising frailty (initial increase of $0.022 \mathrm{eFI}$ per month before slowing from a baseline eFI of 0.21) was associated with a 180\% increase in mortality (OR 2.84, 95\% Cl 2.34-3.45) for $2.2 \%$ of the sample. Moderately increasing frailty (eFl increase of 0.007 per month, with baseline of 0.26 ) was associated with a $65 \%$ increase in mortality (OR $1.65,95 \%$ Cl 1.54-1.76) for $21.2 \%$ of the sample. The largest (76.6\%) class was stable frailty (eFI increase of 0.001 from a baseline of 0.26 ). When cases were reweighted to population level, rapidly rising frailty had $99.1 \%$ specificity and $3.2 \%$ sensitivity (positive predictive value 19.8\%, negative predictive value 93.3\%) for predicting individual risk of mortality.

Conclusions: People aged over 75 with frailty who are at highest risk of death have a distinctive frailty trajectory in the last 12 months of life, with a rapid initial rise from a low baseline, followed by a plateau. Routine measurement of frailty can be useful to support clinicians to identify people with frailty who are potential candidates for palliative care, and allow time for intervention.
\end{abstract}

Keywords: Frailty, Geriatrics, Palliative care, Primary care, End of life

\section{Background}

An increasing number of older people are now dying with a diagnosis of frailty. In high-income countries, the estimated prevalence of frailty is $11 \%$ for people aged over 65 years, rising to $25-50 \%$ for people over 85 [1]. Frailty is characterised by an accumulation of deficits and reduced strength, endurance and physiological function $[2,3]$. It is associated with a range of adverse outcomes, including falls, delirium, disability and mortality [4-8].

\footnotetext{
* Correspondence: barbara.hanratty@newcastle.ac.uk Institute of Health and Society, Newcastle University, Newcastle upon Tyne
} NE2 4AX, UK
Timely recognition of the end-of-life phase is fundamental to the provision of palliative care, as it allows clinicians to introduce generalist or specialist services, to discuss preferences and make advance plans [9-11]. This may be particularly challenging for patients with frailty, where trajectories of decline are gradual and slow, and patients may not have a recognised life-limiting diagnosis $[12,13]$. Despite calls for greater awareness of the benefits of palliative care for people with frailty [14], recognition remains incomplete [15].

Since 2017, general practitioners (GPs) in England have been required to identify and review patients with moderate

(c) The Author(s). 2018 Open Access This article is distributed under the terms of the Creative Commons Attribution 4.0 International License (http://creativecommons.org/licenses/by/4.0/), which permits unrestricted use, distribution, and 
and severe frailty using an appropriate tool. One of the instruments recommended by NHS England is the electronic frailty index (eFI) $[16,17]$. The eFI is a cumulative deficit measure of frailty that uses over 2000 Read codes to calculate a frailty score based on a variety of symptoms, diagnoses and observations recorded in primary care electronic health records [5]. It is available in GP software systems in the UK, and can be calculated automatically from patients' records at any time. Previous research has shown that frailty measured by the eFI at a single time point can predict mortality at a population level [17], but it is not effective at identifying individuals at short-term risk of all-cause mortality before this would be clinically apparent [18]. Frailty is associated with increasing age, but there is also wide inter-individual variation in how deficits are accumulated over time, and studies have demonstrated different longitudinal trajectories of frailty $[19,20]$. However, to date, few studies have examined the association between longitudinal change in frailty and mortality. Where studies have been carried out, they have produced population level results with limited clinical application. For example, recent analysis of data from a US cohort identified distinct frailty trajectories and their associated mortality risk, using scores calculated at annual intervals over 8 years [21].

In this study, we used eFI measured at monthly intervals to examine longitudinal change in frailty in the year before death. The aim was to determine if changes in frailty measured by the eFI could be useful in primary care to indicate increased risk of dying and the need to consider palliative care.

\section{Methods}

\section{Setting}

The study population was sampled by ResearchOne from their health and care research database, containing de-identified clinical and administrative data from approximately six million active electronic healthcare records. ResearchOne extracts anonymised data from the SystmOne clinical information system, which is used in over 35\% (2500) of general practices in England, and holds records on approximately half of the UK population. Primary care professionals use SystmOne to record their consultations (including patient histories, clinical observations, diagnoses, treatments and referrals) with free text and the Read code classification system.

\section{Participants}

ResearchOne identified records of individuals (cases) aged 75 years and over who died between 1 January 2015 and 1 January 2016. A comparison group (controls) was constructed by identifying patients matched $1: 1$ to cases by age, sex and practice location, but with no record of death between 1 January 2015 and 1 January
2016. Patients without eFI scores, those with records available for fewer than 6 months, and those for whom cause of death was classified as an external cause of mortality (accidents, suicides, murders according to International Classification of Diseases codes version 10) were excluded.

\section{Study design}

In this longitudinal study, the relationship between mortality and frailty was determined using eFI scores generated at monthly intervals for 1 year prior to recorded month of death for cases, and 1 year prior to 1 January 2016 for matched controls. Controls were matched at age of death of the case (between 1 January 2015 and 1 January 2016), and sampled on 1 January 2016, when they were known to have survived; hence, controls were, on average, 6 months older than the cases at time of measurement. Approval for this study was granted by the ResearchOne ethical review panel, with oversight from the UK NHS Health Research Authority and the UK Government Health and Social Care Information Centre Confidentiality Advisory Group. ResearchOne was approved by the UK NHS National Research Ethics Service (11/NE/0184).

\section{Measurements}

The eFI is a cumulative deficit measure of frailty that calculates a frailty score based on 36 deficits, drawn from a pool of 2000 clinical Read codes for symptoms, signs, diseases, disabilities and abnormal laboratory test values. An individual's eFI score is calculated by dividing the number of deficits present by the total possible to create a score between zero (no deficits) and one (36 deficits) [17]. In this study, eFI was calculated automatically by ResearchOne at monthly intervals for 1 year, based on the information contained in each participant's clinical record.

\section{Analysis}

Latent growth curve models were used to examine the shape of change in eFI and the relationship between eFI trajectory and age and sex (age mean centred and female as the referent categories). Latent class growth mixture models were used to examine the potential for subgroups (classes) of the population to have different trajectories of change in eFI. The optimum number of classes was determined using sample size-adjusted Bayesian information criterion (BIC) and model entropy. The trajectories estimated from the optimum latent class growth mixture model were used to examine the association between class membership and mortality (case/ control status) as a distal outcome [22]. The longitudinal models were formulated using months as the time variable, with month 0 as baseline and month 12 at death/ 
study end. All longitudinal models were run in Mplus version 8 (Muthén \& Muthén, Los Angeles, CA, USA). To estimate the proportions of the population in each of the trajectory classes, adjusting for the study design (sample of controls), the posterior probabilities for being in each class were reweighted by the population estimates from Office of National Statistics life tables. Weighted class sizes and the association between class membership and mortality were calculated using the Survey package (Lumley, 2017) in $R$ version 3.4.2 ( $R$ Core team, Vienna, Austria).

\section{Results}

This study analysed information on 26,298 individuals (13,149 cases and 13,149 controls), 14,620 (55.6\%) of whom were female and 11,678 (44.4\%) were male. The mean age at death for cases was 85.14 (SD 5.98) years with a pseudo age of 85.66 (SD 5.98) years for controls, as expected by design. Age-adjusted mean eFI at baseline was significantly higher $(p<0.001)$ for cases $(\mathrm{eFI}=0.27$, 9.8 deficits) than for controls (eFI $=0.23,8.3$ deficits), as expected from previous work $[17,18]$, and mean eFI for females (eFI $=0.23,8.3$ deficits) was significantly higher $(p<0.001)$ at baseline than for males (eFI $=0.22,8.0$ deficits) when adjusted for age and case/control status. Table 1 shows the demographic characteristics of the study sample.

The latent growth curve model that best described the shape of frailty change over the study period included freely estimated intercepts, slopes and a quadratic polynomial term for time (see Additional file 1: Table S1 for parameter descriptions and model fit statistics). The estimated mean trajectory of eFI increased by 0.002 (95\% CI 0.001-0.002, 0.1 deficits) per month (at study baseline) from a mean of 0.252 (95\% CI 0.251-0.253, 9.1 deficits).

Table 1 Demographic characteristics of study participants at baseline

\begin{tabular}{lrrrr}
\hline & \multicolumn{1}{l}{ Case } & & Control \\
\hline$n$ & 13,149 & & 13,149 & \\
Female ( $n$, \%) & 7310 & 55.59 & 7310 & 55.59 \\
Male ( $n$, \%) & 5839 & 44.41 & 5839 & 44.41 \\
Age, years (mean, SD) & 85.14 & 5.98 & 85.66 & 5.98 \\
Female age, years (mean, SD) & 86.15 & 6.11 & 86.68 & 6.12 \\
Male age, years (mean, SD) & 83.87 & 5.55 & 84.38 & 5.54 \\
eFl & 27.05 & & 23.27 & \\
Female eFl & 28.08 & & 24.61 & \\
Male eFI & 25.76 & & 21.59 & \\
eFl (age adjusted) & 27.18 & & 23.13 & \\
Female eFI (age adjusted) & 27.87 & & 23.84 & \\
Male eFI (age adjusted) & 26.30 & & 22.27 & \\
\hline
\end{tabular}

The quadratic term was small $(\mathrm{eFI}=0.00003,95 \% \mathrm{CI}$ 0.00003-0.00004) but improved the model fit and was retained to allow flexibility in class structures during the mixture modelling phase. Large variance in the intercept $(\mathrm{eFI}=0.128)$ and slope $(\mathrm{eFI}=0.278)$ was observed, suggesting individual heterogeneity in trajectories. There was some evidence that higher baseline frailty was related to slower frailty increase over time (intercept slope covariance $-0.109, p<0.006$ ).

The quadratic model was extended to investigate the impact of age and sex on the trajectories (Table 2 and Additional file 1: Table S2 for model fit and parameter descriptions). In the full model (Additional file 1: Table S2), eFI at study baseline increased by 0.0047 (95\% CI 0.0044-0.0049, 0.2 deficits; $p<0.001$ ) for each year of age, and men had a mean eFI of $0.0016(95 \%$ CI 0.00133-0.00187, 0.6 deficits; $p<0.001$ ) lower than women. Because there was little evidence that sex or age influenced the trajectory of frailty over time (for each year of age, eFI increased by 0.00002 per month, $95 \% \mathrm{CI}$ $0.00001-0.00003,<0.001$ deficits, $p=0.003$, all other coefficients $p>0.05$ ), the model regressing the intercept only on age and sex, including slopes and quadratic terms (Table 2), was carried forward to investigate potential latent class trajectories.

A three-class latent growth mixture model model (Table 2) was chosen as the most parsimonious with good fit and clinically relevant classes (Additional file 1: Table S3). The most common 'stable' class contained $76.6 \%$ of the sample and showed little change in frailty over time, with eFI increasing by 0.001 per month ( 0.04 deficits, 'stable' class) from a baseline eFI of 0.260 (9.4 deficits). A 'moderate growth' class containing $21.2 \%$ of the sample demonstrated an eFI increase of 0.0045 ( 0.2 deficits) per month from a slightly higher baseline eFI of 0.262 (9.4 deficits), and a small 'rapidly rising' class containing $2.2 \%$ of the sample showed a sharp, distinct increase in eFI of 0.01 per month (0.4 deficits) on average over the year from a lower baseline eFI of 0.206 (7.4 deficits). The majority of individuals in this class accrued deficits quickly, with a $0.02 \mathrm{eFI}$ increase per month over the first 3 months $(\mathrm{s}=0.023,95 \%$ CI $0.021-0.025)$, but the quadratic component $(\mathrm{q}=-0.001$, $95 \% \mathrm{CI}-0.001$ to -0.001 ) indicates that they then experienced a period of relative stability; this group had an average increase in eFI over 1 year 14 times higher than the stable class. Age and sex were associated with baseline frailty $(\mathrm{eFI}=0.005 / 0.2$ deficits per year of age, $\mathrm{eFI}=0.016$ / 0.6 deficits higher for women) across classes, but were only weakly related to class membership (all ORs approximately 1.0).

Compared with the 'stable' class both the 'rapidly rising' class (OR 2.84, 95\% CI 2.34-3.45) and the 'moderate growth' class (OR 1.65, 95\% CI 1.54-1.76) were found to be associated with mortality. Figure 1 displays the mean 
Table 2 Summary of best fitting models for shape of change, the impact of study covariates and class descriptions

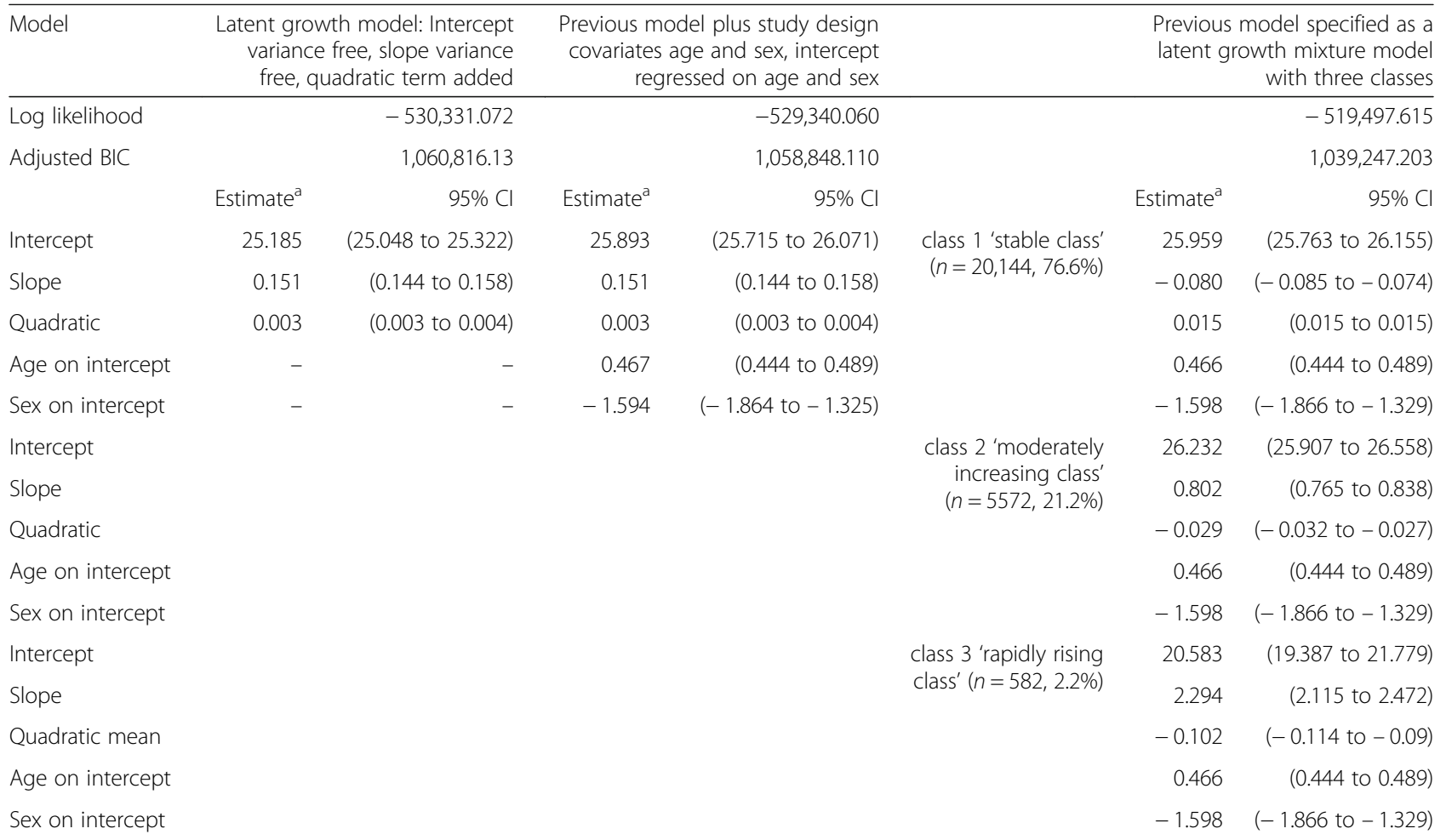

${ }^{a}$ Models used eFI multiplied by 100 to aid estimation and interpretation. Text in results refers to eFI in original units

and observed individual trajectories for each class and the association between class and mortality risk.

To understand the population characteristics, the posterior probabilities for being in each class were reweighted to reflect the general population. Compared with the 'stable class', both the 'rapidly rising' class (OR 3.02, 95\% CI 2.49-3.65) and the 'moderate growth' class (OR 1.58, 95\% CI 1.49-1.67) were associated with an increased chance of mortality. Table 3 contains demographic information for the members of each class.
Reweighting the controls to the population suggests that $1.1 \%$ of the population exhibited rapidly rising frailty; using this group to predict mortality within the year was highly specific (99.1\% specificity), with $19.8 \%$ positive predictive value and $93.3 \%$ negative predictive value, but with low sensitivity (3.2\%). Addition of the 'moderately increasing' class increased the sensitivity (27.8\%) and negative predictive value $(93.9 \%)$ at the expense of a decreased specificity (82.3\%) and positive predictive value (10.4\%).
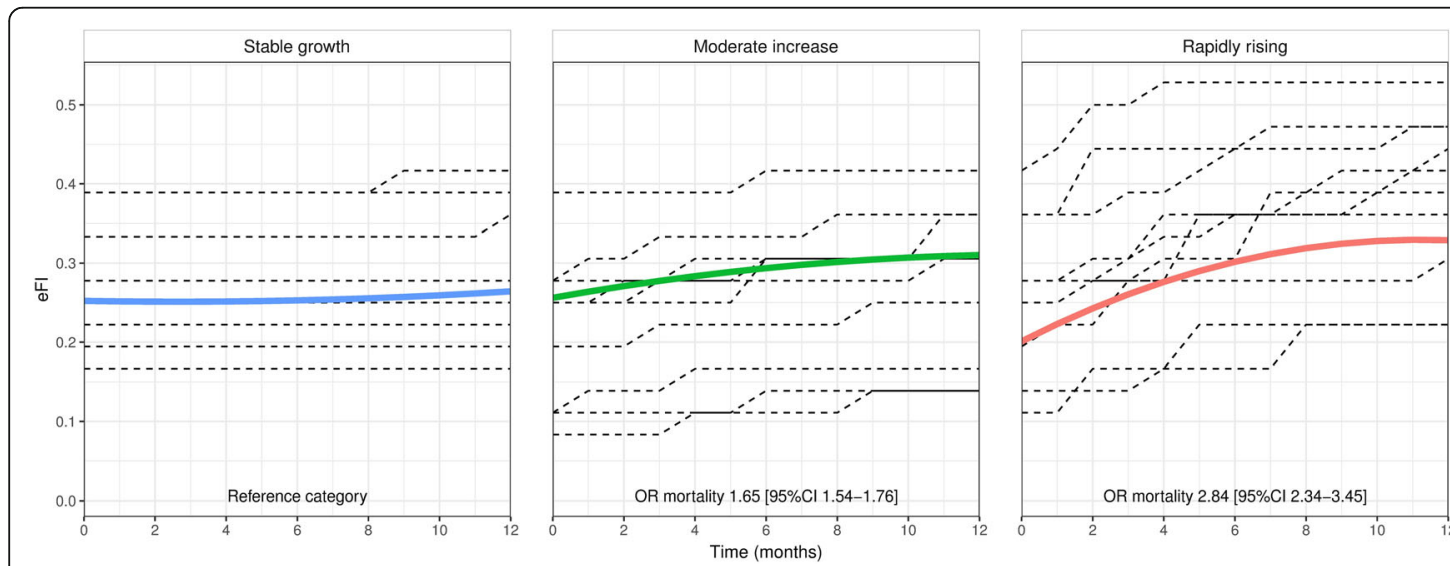

Class estimated means - Rapidly rising - Moderate increase - Stable growth

Fig. 1 Estimated mean trajectories of eFI over 1 year for each of the three latent classes with a random sample of observed individual trajectories for each class 
Table 3 Demographic characteristics of the classes and the proportion of individuals in each class from the age- and sex-adjusted model reweighted to the population level

\begin{tabular}{|c|c|c|c|c|c|c|c|c|c|}
\hline & $n$ & $\%$ & $n$ Female & $\%$ Female & Reweighted $n^{a}$ & $\%$ & Mean age & SD & Mean eFl \\
\hline Total & 26,298 & - & 14,620 & 55.59 & 191,584 & - & - & - & - \\
\hline Rapidly rising & 583 & 2.22 & 327 & 56.09 & 2100 & 1.10 & 85.81 & 5.80 & 0.21 \\
\hline Moderate growth & 5571 & 21.18 & 3105 & 55.74 & 33,225 & 17.34 & 85.57 & 5.88 & 0.26 \\
\hline Stable & 20,144 & 76.60 & 11,188 & 55.54 & 156,259 & 81.56 & 85.34 & 6.02 & 0.25 \\
\hline Cases total & 13,149 & - & 7310 & 55.59 & 13,149 & 6.86 & - & - & - \\
\hline Rapidly rising & 415 & 3.16 & 241 & 58.07 & 415 & 3.16 & 85.13 & 5.92 & 0.23 \\
\hline Moderate growth & 3242 & 24.66 & 1743 & 53.76 & 3242 & 24.66 & 85.21 & 5.90 & 0.28 \\
\hline Stable & 9492 & 72.19 & 5326 & 56.11 & 9492 & 72.19 & 85.11 & 6.01 & 0.27 \\
\hline Controls total & 13,149 & - & 7310 & 55.59 & 178,435 & 93.14 & - & - & - \\
\hline Rapidly rising & 167 & 1.27 & 86 & 51.50 & 1685 & 0.94 & 87.45 & 5.17 & 0.18 \\
\hline Moderate growth & 2330 & 17.72 & 1362 & 58.45 & 29,983 & 16.80 & 86.08 & 5.82 & 0.24 \\
\hline Stable & 10,652 & 81.01 & 5862 & 55.03 & 146,767 & 82.25 & 85.54 & 6.01 & 0.23 \\
\hline
\end{tabular}

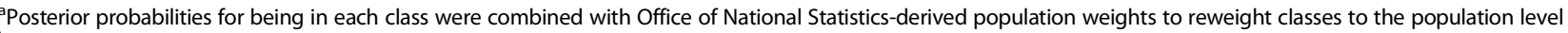

${ }^{\mathrm{b}}$ Age adjusted

\section{Discussion}

We have identified a distinct frailty trajectory that is associated with an increased risk of mortality amongst older adults. Individuals with rapidly rising frailty (average monthly rise in eFI of 0.01 or accrual of 0.4 deficits) are twice as likely to die within 12 months as individuals with stable frailty. Individuals in this class experience an initial monthly increase in eFI of 0.02 , prior to slowing around 8-9 months prior to death. This rapidly rising class represents a small but important proportion of the population who may not be recognised as being at high risk of death, as they started from a lower mean baseline eFI than the rest of the population [17]. The 'moderately increasing frailty' class are also at a greater risk of mortality, with an average rise of 0.005 in eFI per month associated with a $58 \%$ increase in mortality.

Our study demonstrated that, for a small proportion of the population, significant changes in frailty occur over 1 year. Individuals with lower levels of frailty who experience a rapid rise have a greater risk of death over 1 year than individuals with higher, but stable frailty.

The association between severity of frailty measured at a single time point and risk of mortality has been robustly demonstrated at the population level [23]. However, our previous work found that this association did not translate into a useful measure for predicting individual risk of mortality when frailty is measured at a single time point, even close to death [18]. Building on previous work that has examined the relationship between longitudinal change in frailty and mortality at yearly intervals $[21,24]$, we have been able to measure frailty changes at monthly intervals over the final year of life. Our findings suggest that some individuals are resilient to higher but stable levels of frailty, and that changes in frailty status may be a more useful indicator of mortality risk. The positive predictive value and sensitivity for the rapidly rising trajectory mean that recognition of this trajectory alone may not be a reliable predictor of imminent death. However, it may be useful to support clinical judgement and as a risk stratification tool, especially as many individuals who follow this trajectory may not be considered as 'at risk' because of their low levels of baseline frailty. The Q Mortality risk prediction algorithm, which incorporates a frailty index and was designed specifically to assess mortality risk, has better cross-sectional prediction of mortality than the eFI. However, the frailty index implemented in $\mathrm{Q}$ mortality does not currently include a weighting for risk based on changes in frailty scores over time [25].

\section{Strengths and limitations}

We have presented a large population-based longitudinal study of change in frailty measured with the eFI at monthly intervals. Our analysis used a measure of frailty that is available to the majority of general practitioners in England, enhancing the potential for the findings to be applied in practice. The trajectories have potential relevance to older populations in other countries, particularly when frailty is measured using a cumulative deficit approach. Much previously published work on frailty has used data from cohort studies, subject to all the potential limitations of observational research. In this study, participants were drawn from a large, unselected primary care cohort, demographically and geographically representative of England's population, and data were routinely collected.

However, there were limitations to our approach. First, the design as implemented at data extraction meant 
controls were identified at the end of the study (1 January 2016), then matched to individuals who had died in the preceding year (matching was at date of death). Follow-up for the controls was determined from 1 January 2015 to 1 January 2016, but for the cases, follow-up was calculated for 1 year prior to date of death. Controls were not chosen at the point of case death, so this created an imbalance in ages between cases and controls whereby controls were, on average, 6 months older than cases. However, all analyses were adjusted for age, and as frailty increases with age, controls are more likely to have been measured at a higher point in their frailty trajectory and therefore would attenuate our findings if any residual effect of age remained.

Secondly, because study exclusion criteria were applied by ResearchOne at the time of data extraction, we do not have information about the number of people who were not eligible for inclusion in our analysis. Electronic health record systems also rely on clinicians recording their observations correctly using the Read code system, and we were unable to make any assessment of patterns of incorrect or differential coding; nevertheless, this is the same information as used for clinical decisions.

Finally, the way in which eFI is calculated and the data on which it is based (mostly long-term or irreversible deficits), means that eFI scores can only increase or remain stable. This means that any analysis using eFI would not be able to capture improvement or recovery in frailty, which is known to be feasible with interventions such as physical exercise [26-28].

\section{Conclusions}

This longitudinal population-based study demonstrates that it is possible to use a frailty index calculated within electronic healthcare records to identify people who are at a higher risk of dying within 1 year. This has potential application in health services to support clinicians in identifying older adults dying with frailty who may have been overlooked by traditional approaches and to help ensure appropriate care is offered. The rapid initial rise in eFI from a low baseline, followed by a plateau, may be particularly useful in primary care, promoting recognition of people at risk of death, but also allowing time for intervention.

\section{Additional file}

Additional file 1: Table S1. Model development latent growth curve model fit and parameters to determine model shape*. Table S2. Model description and goodness-of-fit statistics for latent growth curves including age and sex*. Table S3. Full description of latent growth mixture parameter characteristics and model fits*. (DOCX $41 \mathrm{~kb}$ )

\section{Abbreviations}

BIC: Bayesian information criterion; Cl: Confidence interval; eFI: Electronic frailty index; GP: General practitioner; NHS: National Health Service; OR: Odds ratio; SD: Standard deviation; UK: United Kingdom

\section{Acknowledgements}

Members of VoiceNorth (a Newcastle University-funded organisation that aims to capture the public's views on research and policy developments) were involved in this research. A number of them had been caregivers for a relative or friend at the end of life. Their views helped to shape the research question, and will help us to develop a plain language summary for wider dissemination

\section{Funding}

This work was supported by the National Institute for Health Research (NIHR) School for Primary Care Research (SPCR, 2016DS to DS) and the Medical Research Council (MC_U105292687 to FEM). The views expressed are those of the authors and not necessarily those of the NHS, the NIHR or the Department of Health. The funders had no role in study design, data collection and analysis, decision to publish, or preparation of the manuscript.

\section{Availability of data and materials}

The data that support the findings of this study are available from ResearchOne, but restrictions apply to the availability of these data, which were used with appropriate permissions for the current study, and so are not publicly available. Data are not available from the authors because they relate to real and current NHS health records, and the authors do not have permission to share these data.

\section{Authors' contributions}

$\mathrm{BH}$ and FEM designed the study and obtained the data, DS undertook the analysis supervised by FEM and wrote the first draft of the paper, all authors edited the paper and agree with the text. DS is guarantor of the analysis.

\section{Ethics approval and consent to participate}

Approval for this study was granted by the ResearchOne ethical review panel with oversight from the UK NHS Health Research Authority and the UK Government Health and Social Care Information Centre Confidentiality Advisory Group. ResearchOne was approved by the UK National Health Service (NHS) National Research Ethics Service (11/NE/0184).

Consent for publication

Not applicable.

\section{Competing interests}

The authors declare that they have no competing interests.

\section{Publisher's Note}

Springer Nature remains neutral with regard to jurisdictional claims in published maps and institutional affiliations.

Received: 30 April 2018 Accepted: 6 August 2018

Published online: 21 September 2018

\section{References}

1. Collard RM, Boter H, Schoevers RA, Oude Voshaar RC. Prevalence of frailty in community-dwelling older persons: a systematic review. J Am Geriatr Soc. 2012;60(8):1487-92

2. Clegg A, Young J, lliffe S, Rikkert MO, Rockwood K. Frailty in elderly people. Lancet. 2013;381(9868):752-62.

3. Morley JE, Vellas B, Abellan van Kan G, Anker SD, Bauer JM, Bernabei R, et al. Frailty consensus: a call to action. J Am Med Dir Assoc. 2013:14(6):392-7.

4. Fried LP, Tangen CM, Walston J, Newman AB, Hirsch C, Gottdiener J, et al. Frailty in older adults: evidence for a phenotype. J Gerontol A Biol Sci Med Sci. 2001;56(3):M146-56.

5. Rockwood K, Mitnitski A. Frailty in relation to the accumulation of deficits. J Gerontol A Biol Sci Med Sci. 2007;62(7):722-7.

6. Theou O, Brothers TD, Mitnitski A, Rockwood K. Operationalization of frailty using eight commonly used scales and comparison of their ability to predict all-cause mortality. J Am Geriatr Soc. 2013;61(9):1537-51.

7. Drubbel I, de Wit NJ, Bleijenberg N, Eijkemans RJ, Schuurmans MJ, Numans ME. Prediction of adverse health outcomes in older people using a frailty index based on routine primary care data. J Gerontol A Biol Sci Med Sci. 2013;68(3):301-8. 
8. Hope AA, Gong MN, Guerra C, Wunsch H. Frailty before critical illness and mortality for elderly Medicare beneficiaries. J Am Geriatr Soc. 2015;63(6): $1121-8$.

9. Jeng G, Tinetti ME. Changes in end-of-life care. JAMA. 2015;309:489-90.

10. Eynon T, Lakhani MK, Baker R. Never the right time: advance care planning with frail and older people. Br J Gen Pract. 2013;63:511-2.

11. National Institute for Health and Care Excellence. NICE Quality Standard 13: End of Life Care for Adults. London: NICE; 2017.

12. Dixon J, King D, Matosevic T, Clark M, Knapp M. Equity in the Provision of Palliative Care in the UK: Review of Evidence. Personal Social Services Research Unit: London School of Economics. 2015. https://www.pssru.ac.uk/ pub/4962.pdf. Accessed 1 Feb 2018

13. Cohen-Mansfield J, Skornick-Bouchbinder M, Brill S. Trajectories of end of life: a systematic review. J Gerontol B. 2018;73(4);564-72.

14. Cardona-Morrell M, Lewis E, Suman S, Haywood C, Williams M, Brousseau $\mathrm{AA}$, et al. Recognising older frail patients near the end of life: what next? Eur J Intern Med. 2017:45:84-90.

15. Tapsfield J, Hall C, Lunan C, McCutcheon H, McLoughlin P, Rhee J, et al. Many people in Scotland now benefit from anticipatory care before they die: an after death analysis and interviews with general practitioners. BMJ Support Palliat Care. 2016;(0):1-10. https://doi.org/10.1136/bmjspcare-2015001014

16. Moody D, Lyndon H, Stevens G. Toolkit for General Practice in Supporting Older People Living with Frailty. Leeds: NHS England; 2017.

17. Clegg A, Bates C, Young J, Ryan R, Nichols L, Ann Teale E, et al. Development and validation of an electronic frailty index using routine primary care electronic health record data. Age Ageing. 2016;45(3):353-60.

18. Stow D, Matthews FE, Barclay S, lliffe S, Clegg A, De Biase S, et al. Evaluating frailty scores to predict mortality in older adults using data from population based electronic health records: case control study. Age Ageing. 2018;47(4); 564-69.

19. Hsu HC, Chang WC. Trajectories of frailty and related factors of the older people in Taiwan. Exp Aging Res. 2015;41(1):104-14.

20. Marshall A, Nazroo J, Tampubolon G, Vanhoutte B. Cohort differences in the levels and trajectories of frailty among older people in England. J Epidemiol Community Health. 2015;69:316-21.

21. Chamberlain AM, Finney Rutten $\sqcup$, Manemann SM, Yawn BP, Jacobson DJ, Fan C, et al. Frailty trajectories in an elderly population-based cohort. J Am Geriatr Soc. 2016;64(2):285-92.

22. Lanza ST, Tan X, Bray BC. Latent class analysis with distal outcomes: a flexible model-based approach. Struct Equ Modeling. 2013;20(1):1-26.

23. Kojima $G$, lliffe $S$, Walters $K$. Frailty index as a predictor of mortality: a systematic review and meta-analysis. Age Ageing. 2018;47(2):193-200.

24. Buchman AS, Wilson RS, Bienias JL, Bennett DA. Change in frailty and risk of death in older persons. Exp Aging Res. 2009;35(1):61-82.

25. Hippisley-Cox J, Coupland C. Development and validation of QMortality risk prediction algorithm to estimate short term risk of death and assess frailty: cohort study. BMJ. 2017;358:j4208.

26. Clegg AP, Barber SE, Young JB, Forster A, lliffe SJ. Do home-based exercise interventions improve outcomes for frail older people? Findings from a systematic review. Rev Clin Gerontol. 2012;22(1):68-78.

27. Puts MTE, Toubasi S, Andrew MK, Ashe MC, Ploeg J, Atkinson E, et al. Interventions to prevent or reduce the level of frailty in communitydwelling older adults: a scoping review of the literature and international policies. Age Ageing. 2017:46(3):383-92.

28. Rogers NT, Marshall A, Roberts CH, Demakakos P, Steptoe A, Scholes S. Physical activity and trajectories of frailty among older adults: evidence from the English longitudinal study of ageing. PLoS One. 2017;12(2):e0170878.

\section{Ready to submit your research? Choose BMC and benefit from:}

- fast, convenient online submission

- thorough peer review by experienced researchers in your field

- rapid publication on acceptance

- support for research data, including large and complex data types

- gold Open Access which fosters wider collaboration and increased citations

- maximum visibility for your research: over $100 \mathrm{M}$ website views per year

At BMC, research is always in progress.

Learn more biomedcentral.com/submissions 